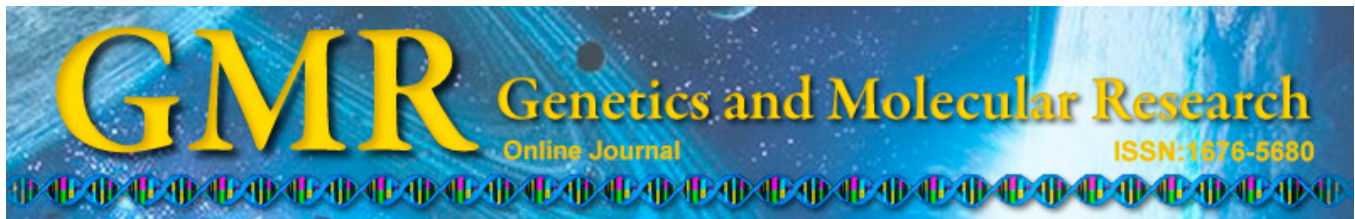

\title{
Genetic diversity and population genetic structure of the miiuy croaker, Miichthys miiuy, in the East China Sea by microsatellite markers
}

\author{
Y. Qin, D.Q. Sun, T.J. Xu, X.Z. Liu and Y.N. Sun \\ Laboratory of Fish Biogenetics \& Immune Evolution, College of Marine Science, \\ Zhejiang Ocean University, Zhoushan, China \\ Corresponding authors: Y.N. Sun / X.Z. Liu \\ E-mail: Yuenasun@163.com / xuem07@163.com
}

Genet. Mol. Res. 13 (4): 10600-10606 (2014)

Received June 6, 2014

Accepted December 9, 2014

Published December 18, 2014

DOI http://dx.doi.org/10.4238/2014.December.18.1

\begin{abstract}
Genetic diversity and patterns of population structure of the miiuy croaker were investigated using SSR markers. A set of 10 microsatellite loci revealed 40 alleles; the number of alleles varied from 2 to 10 for each marker. A relatively high level of genetic variability was observed between miiuy croaker individuals. Genetic diversity was relatively high within populations with corresponding high average gene flow. There were genealogical branches or clusters corresponding to sampling localities according to the UPGMA tree and principal component analysis. Knowledge of the genetic diversity and population structure will be crucial for establishing appropriate fishery management stocks for this species.
\end{abstract}

Key words: Miiuy croaker; Microsatellite; Genetic diversity; Population structure 


\section{INTRODUCTION}

The miiuy croaker, Miichthys miiuy, is distributed throughout eastern China (Zhang and Hong, 2000; Shan et al., 2008a,b). It is a very important food fish species due to its good taste and high nutritive value (Sun et al., 2005; Peng et al., 2010). This species inhabits coastal waters and spawns in autumn from September to November (Li et al., 2005). However, the resources of the miiuy croaker have declined due to overfishing, pollution, coastal construction, and other factors (An et al., 2012). At the same time, high mortality and poor growth have been frequently encountered during artificial larval rearing, which hinders the mass production of this fish (Shan et al., 2009).

In recent years, most studies on the miiuy croaker were about morphological and physiological characteristics and molecular immunology (Liu et al., 2007; Cheng et al., 2011a,b; Xu et al., 2011a,b; Meng et al., 2012; Sun et al., 2005, 2012). However, little is known about the genetics of the miiuy croaker. Patterns of genetic diversity of individuals and populations could provide an important basis for ecological restoration measures (Väli et al., 2008), as well as the protection of germplasm resources. The identification of fish populations is an important step towards establishing conservation policies that can protect locally adapted populations by regulating fishing activities (Carvalho and Hauser, 1994).

As we all know, molecular genetic techniques can offer the ability to identify and delineate fish stock structure. Microsatellite markers have been considered some of the most efficient molecular markers, providing abundant genetic information due to their co-dominance, high mutation rate, abundance throughout the genome and ease of scoring. They could be used to analyze the genetic diversity and population structure of the miiuy croaker. In this study, about thirty individuals per population were collected from six regions of the East China Sea. We then used 10 microsatellite markers to investigate genetic diversity and population structure. This information could provide an important theoretical basis for the protection and sustainable utilization of this species.

\section{MATERIAL AND METHODS}

\section{Sample collection, DNA extraction and SSR analysis}

An average of thirty wild miiuy croaker individuals per population were collected from the following six geographic regions along the coast of the East China Sea: Zhoushan, Xiangshan, Wenling, Wenzhou, Yueqing, and Ruian (Figure 1). Fin tissues were dissected from each individual and immediately preserved in pure alcohol and stored at $-20^{\circ} \mathrm{C}$. Genomic DNA was extracted from the fin clips using the traditional phenol-chloroform method with some modifications. The quality and quantity of DNA were examined on agarose gel electrophoresis. DNA was finally adjusted to $100 \mathrm{ng} / \mu \mathrm{L}$ and stored at $4^{\circ} \mathrm{C}$ for later use.

Ten polymorphic SSR loci from our library were used in this study (Wang et al., 2010; $\mathrm{Xu}$ et al., 2011c). PCR was carried out in a $15-\mu \mathrm{L}$ reaction mixture containing $9.9 \mu \mathrm{L}$ sterile water, $1.5 \mu \mathrm{L}$ 10X PCR buffer (containing $1.5 \mathrm{mM} \mathrm{Mg}^{2+}$ ), $1.2 \mu \mathrm{L} 2.5 \mathrm{mM}$ dNTPs, $0.6 \mu \mathrm{L}$ forward primer, $0.6 \mu \mathrm{L}$ reverse primer, $1 \mu \mathrm{L}$ diluted DNA template, and $0.1 \mu \mathrm{L} 5 \mathrm{U} / \mu \mathrm{L}$ Taq DNA polymerase (Tiangen). PCR amplification was performed on an ABI 9700 according to the following profile: $95^{\circ} \mathrm{C}$ for $5 \mathrm{~min} ; 30$ cycles of $95^{\circ} \mathrm{C}$ for $30 \mathrm{~s}$, annealing temperature for $30 \mathrm{~s}$ and $72^{\circ} \mathrm{C}$ for $30 \mathrm{~s}$; and $72^{\circ} \mathrm{C}$ for $5 \mathrm{~min}$. The amplified products were denatured for 8 min at $96^{\circ} \mathrm{C}$. The denatured products were separated on $6 \%$ denaturing polyacrylamide (19:1 
acrylamide:bis-acrylamide) gels and visualized by silver staining. Denatured pBR322 DNA/ MspI molecular weight marker (Tiangen) was used as the size standard to determine size of PCR products.

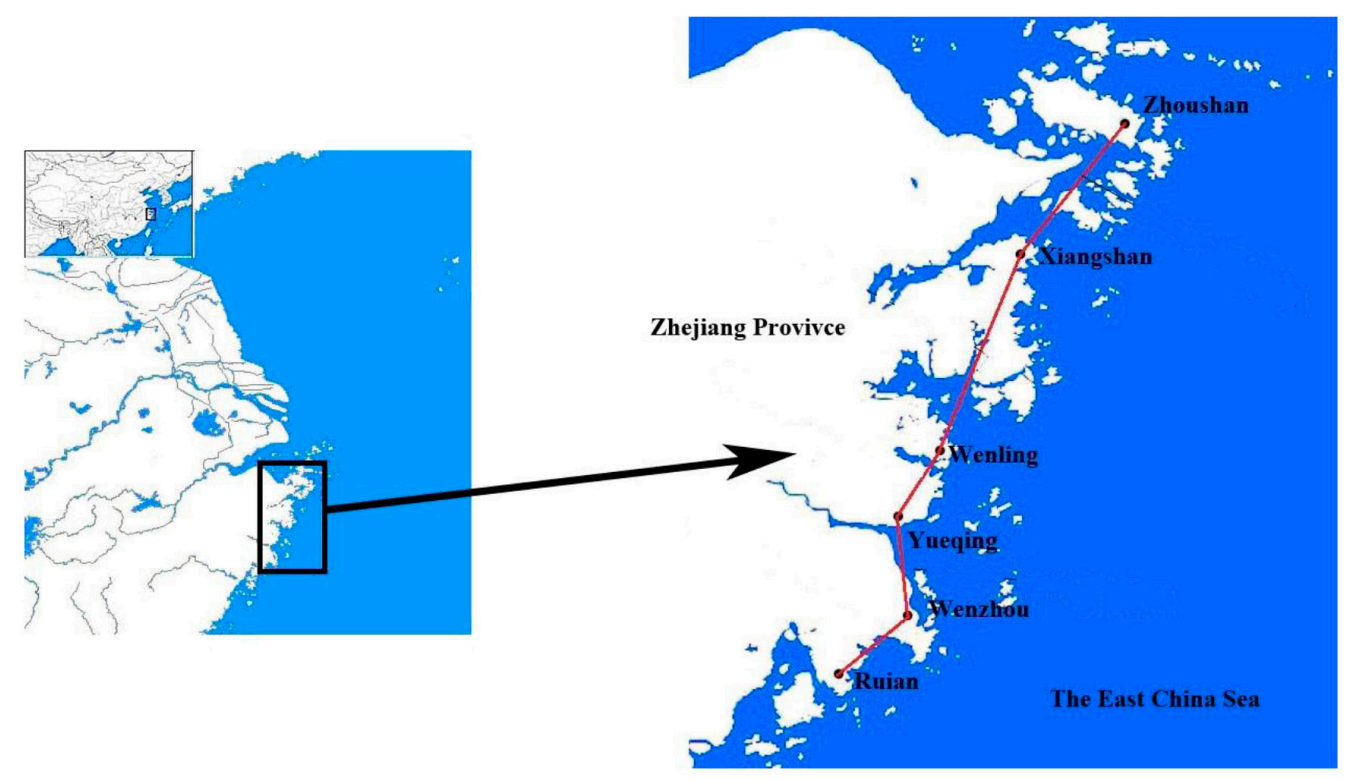

Figure 1. Sample locations for the miiuy croaker.

\section{Data analysis}

For the six populations, percentage of polymorphic loci, observed number of alleles $\left(N_{\mathrm{A}}\right)$, effective number of alleles $\left(N_{\mathrm{E}}\right)$, observed heterozygosity $\left(H_{\mathrm{O}}\right)$, expected heterozygosity $\left(H_{\mathrm{E}}\right)$, Shannon's information index $(I)$, Nei's genetic diversity, and genetic distance were calculated by POPGENE version 1.32 (Yeh and Yang, 1997). Molecular variances within and between six populations were estimated by analysis of molecular variance (AMOVA) using ARLEQUIN 3.0 (Excoffier et al., 2005). The distribution of populations was presented using principal coordinate analysis (PCA) (Gower, 1966). MVSP (Multivariate Statistical Package ver. 3.1) software was used to construct PCA graphs the (Kovach, 1999).

\section{RESULTS}

\section{SSR polymorphism and genetic variation}

The 10 loci were all polymorphic in the populations studied. The detail of polymorphism is given in Table 1 . The average $H_{\mathrm{O}}$ was 0.31 and average $H_{\mathrm{E}}$ was 0.43 . The hierarchical F-statistics estimated according to Wright (1965), ranged from 0.03 to 0.14 , with an average of 0.08 . A total of 40 observed alleles were detected. $N_{\mathrm{E}}$ per locus ranged from 1.18 to 7.52 . 
Table 1. Genetic differentiation and the gene flow among populations of miiuy croaker.

\begin{tabular}{lrccccccccc}
\hline Locus & $N_{\mathrm{A}}$ & $N_{\mathrm{E}}$ & $N_{\mathrm{m}}$ & $H_{\mathrm{O}}$ & $H_{\mathrm{E}}$ & $F_{\text {IS }}$ & $F_{\text {IT }}$ & $F_{\text {ST }}$ & PIC & $I$ \\
\hline Mimi-1 & 4 & 1.85 & 6.71 & 0.47 & 0.48 & 0.10 & 0.06 & 0.04 & 0.39 & 0.78 \\
Mimi-19 & 3 & 1.25 & 3.38 & 0.15 & 0.20 & 0.20 & 0.26 & 0.07 & 0.19 & 0.42 \\
Mimi-24 & 3 & 1.46 & 1.53 & 0.11 & 0.32 & 0.66 & 0.71 & 0.14 & 0.29 & 0.59 \\
Mimi-28 & 3 & 1.38 & 1.68 & 0.19 & 0.28 & 0.08 & 0.20 & 0.13 & 0.25 & 0.52 \\
Mimi-16-E10 & 3 & 1.66 & 7.43 & 0.38 & 0.40 & 0.04 & 0.01 & 0.03 & 0.33 & 0.64 \\
Mimi-56-G06 & 6 & 2.84 & 7 & 0.48 & 0.65 & 0.19 & 0.28 & 0.12 & 0.61 \\
Mimi-43-H04 & 10 & 7.52 & 2.98 & 0.54 & 0.87 & 0.29 & 0.35 & 0.08 & 0.85 & 2.13 \\
Mimi-13-G10 & 3 & 2.36 & 9.52 & 0.46 & 0.58 & 0.13 & 0.16 & 0.03 & 0.49 & 0.94 \\
Mimi-21-G10 & 2 & 1.18 & 2.07 & 0.07 & 0.15 & 0.40 & 0.46 & 0.11 & 0.14 & 0.289 \\
Mimi-42-G06 & 3 & 1.49 & 2.92 & 0.23 & 0.33 & 0.22 & 0.28 & 0.08 & 0.29 & 0.60 \\
Mean & 4 & 2.30 & 4.52 & 0.31 & 0.43 & 0.23 & 0.27 & 0.08 & 0.38 & 0.82 \\
\hline
\end{tabular}

$N_{\mathrm{A}}=$ observed number of alleles; $N_{\mathrm{E}}=$ effective number of alleles; $N_{\mathrm{m}}=$ gene flow; $H_{\mathrm{O}}=$ observed heterozygosity; $H_{\mathrm{E}}=$ expected heterozygosity; $F_{\mathrm{IS}}=$ within-population inbreeding coefficient; $F_{\mathrm{IT}}=$ among-population inbreeding coefficient; $F_{\mathrm{ST}}=$ F-statistic, $N_{\mathrm{m}}=0.25\left(1-F_{\mathrm{ST}}\right) / F_{\mathrm{ST}}$; PIC = polymorphism information content; $I=$ the Shannon's information index.

For the six populations, the average polymorphism information content per locus ranged from 0.14 to 0.85 . The average Shannon index $(I)$ was 0.8230 for each locus. Values of gene flow at each locus varied with a range of 1.53 to 9.52 . Analysis of genetic variation between populations of miiuy croaker based on 10 SSR loci is shown in Table 2. $N_{\mathrm{A}}$ was from 2.5 to 3.5 and $N_{\mathrm{E}}$ was from 1.81 to 2.37 . AMOVA analysis suggested that genetic variation occurred mainly within individuals (71.97\%), while that between populations was only $28.03 \%$ (Table 3).

Table 2. Details on effective number of alleles per population $\left(N_{\mathrm{E}}\right)$; observed number of alleles $\left(N_{\mathrm{A}}\right)$; and Shannon's information index $(I)$ of genetic variation within populations of miiuy croaker.

\begin{tabular}{llll}
\hline Population & $N_{\mathrm{A}}$ & $N_{\mathrm{E}}$ & $I$ \\
\hline Zhoushan & 3.5 & 2.37 & 0.85 \\
Wenling & 3.2 & 1.81 & 0.66 \\
Wenzhou & 3.4 & 2.18 & 0.73 \\
Yueqing & 3.0 & 2.04 & 0.83 \\
Xiangshan & 2.5 & 1.92 & 0.58 \\
Ruian & 2.6 & 1.98 & 0.64 \\
\hline
\end{tabular}

Table 3. Analysis of molecular variance (AMOVA) among and within the six populations.

\begin{tabular}{lrccccc}
\hline Source of variation & d.f. & Sum of squares & Variation components & Percentage of variation & $F$ statistic & P \\
\hline Among populations & 5 & 20.50 & 0.063 & 28.02 & 0.03 \\
Within populations & 89 & 173.17 & 0.53 & 71.97 & $<0.01$ \\
Total & 94 & 193.67 & 0.60 & & \\
\hline
\end{tabular}

\section{Population structure}

Data only from the polymorphic SSR loci were analyzed by MVSP. PCA results carried out with MVSP are shown in Figure 2. We constructed a two-dimensional scatter plot for the first two principal factors for the six miiuy croaker populations. We found that the Yueqing and Wenzhou populations could be in one cluster, and the other four populations could be in 
another cluster. The genetic distance between the six populations showed remarkable differences, ranging from 0.03 to 0.10 (Table 4).

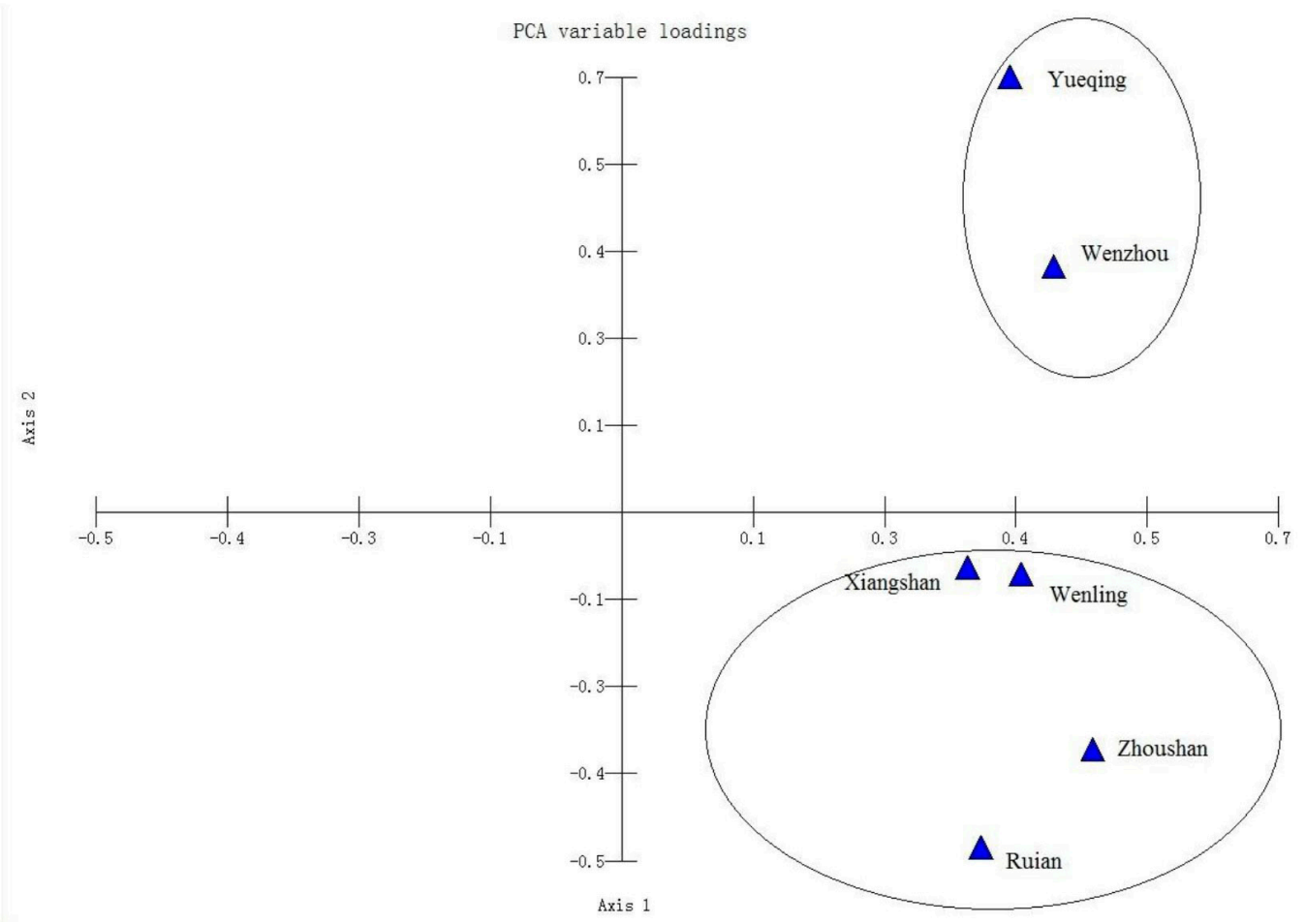

Figure 2. Two-dimensional scatter plot for the first two principal factors for six miiuy croaker populations.

Table 4. Nei's genetic distance of the six populations.

\begin{tabular}{lccccc}
\hline & Zhoushan & Wenling & Wenzhou & Yueqing & Xiangshan \\
\hline Zhoushan & - & & & & \\
Wenling & 0.0517 & - & & & \\
Wenzhou & 0.0415 & 0.0276 & - & - & - \\
Yueqing & 0.0827 & 0.0888 & 0.0376 & 0.0685 & 0.0488 \\
Xiangshan & 0.0563 & 0.0650 & 0.0557 & 0.1014 & - \\
Ruian & 0.0780 & 0.0764 & 0.0687 & & \\
\hline
\end{tabular}

\section{DISCUSSION}

Population genetic structure of a species can provide critical information for developing conservation and management strategies. In this study, we provided new evidence of genetic diversity for the miiuy croaker. Marine migratory fishes with high dispersal capabilities and large effective population sizes are anticipated to show high levels of gene flow and a low degree of differentiation (Beheregaray and Sunnucks, 2001). AMOVA showed that the 
molecular variations observed in the miiuy croaker mainly occurred within the populations, which may suggest a weak and unstable regional genetic structure in this species. Such population structuring could indicate that gene flow is reduced between these regions.

Heterozygosity is an important index for population variation at the genetic level; $H_{\mathrm{O}}$ could be easily influenced by sample size, so $H_{\mathrm{E}}$ can reflect the genetic diversity better. The average $H_{\mathrm{E}}$ in this study at the population level was 0.43 . It showed that genetic diversity in the species was relatively high. In addition, the average PIC ( 0.38$)$ also proved the same result. The species with a relatively high average $N_{\mathrm{E}}$ suggests that it has good genetic variation and could be amenable to protection of germplasm resources, while there should be concern for the population with a lower genetic differentiation with regard to enhancing protection. In this research, the average $N_{\mathrm{E}}$ was 2.30 , so we can conclude that this species has a relatively steady genetic diversity. Genetic diversity is closely related to adaptive power, viability and evolutionary potential. Finally, it also showed that this species has the genetic potential for breeding.

Genetic diversity and molecular systematic data can contribute to the development of effective conservation strategies. The genetic data obtained here for the miiuy croaker based on microsatellite markers demonstrated indirectly the adaptive genetic diversity. These data provide genetic information for each population, so that a great amount of genetic variation in the miiuy croaker can be preserved.

\title{
ACKNOWLEDGMENTS
}

\author{
Research supported by Zhejiang Province Natural Science Foundation (\#LY13C040001 \\ and \#LY13C040001).
}

\section{REFERENCES}

An HS, Kim EM, Lee JW, Kim DJ, et al. (2012). New polymorphic microsatellite markers in the Korean miiuy croaker, Miichthys miiuy, and their application to the genetic characterization of wild and farmed populations. Anim. Cell. Sys. 16: 41-49.

Beheregaray LB and Sunnucks P (2001). Fine-scale genetic structure, estuarine colonization and incipient speciation in marine silverside fish Odontesthes argentinensis. Mol. Ecol. 10: 2849-2866.

Carvalho GR and Hauser L (1994). Molecular genetics and the stock concept in fisheries. Rev. Fish. Biol. Fish. 4: 326-350.

Cheng YZ, Wang RX, Sun YN and Xu TJ (2011a). Molecular characterization of miiuy croaker CC chemokine gene and its expression following Vibrio anguillarum injection. Fish Shellfish Immunol. 31: 148-154.

Cheng YZ, Wang RX and Xu TJ (2011b). Molecular cloning, characterization and expression analysis of a miiuy croaker (Miichthys miiuy) CXC chemokine gene resembling the CXCL9/CXCL10/CXCL11. Fish Shellfish Immunol. 31: 439-445.

Excoffier L, Laval G and Schneider S (2005). Arlequin (version 3.0): An integrated software package for population genetics data analysis. Evol. Bioinform. 1: 47-55.

Gower JC (1966). Some distance properties of latent root and vector methods used in multivariate analysis. Biometrika 55: $582-585$.

Kovach W (1999). MVSP-A Multivariate Statistical Package for Windows. Ver. 3.1. Kovach Computing Services, Pentraeth, Wales, 133.

Li MY, Zheng ZM and Zhu JQ (2005). Bloodstock culture and artificial propagation of Miichthys miiuy (Basilewsky). J. Fish. Sci. China 24: 32-34.

Liu MH, Luo HZ, Chen B, Peng ZL, et al. (2007). Effects of $\mathrm{Cu}^{2+}$ and $\mathrm{Cd}^{2+}$ on $\mathrm{Na}^{+}-\mathrm{K}^{+}$-ATPase activity of gills and superoxide dismutase activity of liver in Miichthys miiuy. J. Safety Environ. 7: 5-8.

Meng FX, Wang RX, Xu TJ, Sun YN, et al. (2012). An unexpected loss of domains in the conservative evolution ninth complement component in a higher teleost, Miichthys miiuy. Fish Shellfish Immunol. 32: 1171-1178.

Peng ZL, Liu MH, Fu RB, Luo HZ, et al. (2010). Comparative studies on the molecular genetic diversities between the Zhoushan wild Miichthys miiuy and their early filial generation by AFLP markers. J. Shanghai. Ocean Univ. 19: $172-177$.

Genetics and Molecular Research 13 (4): 10600-10606 (2014)

CFUNPEC-RP www.funpecrp.com.br 
Shan XJ, Cao L, Huang W and Dou SZ (2008a). Feeding, morphological changes and allometric growth during starvation in miiuy croaker larvae. Environ. Biol. Fish. 86: 121-130.

Shan XJ, Xiao ZZ, Huang W and Dou SZ (2008b). Effects of photoperiod on growth, mortality and digestive enzymes in miiuy croaker larvae and juveniles. Aquaculture 281: 70-76.

Shan XJ, Huang W, Cao L and Xiao ZZ (2009). Ontogenetic development of digestive enzymes and effect of starvation in miiuy croaker Miichthys miiuy larvae. Fish. Physiol. Biochem. 35: 385-398.

Sun QH, Shi WD and Sun JZ (2005). Morphological studies on the early development of Miichthys miiuy. S. China Fish. Sci. 6: 8-17.

Sun YY, Zhu ZH, Wang RX, Sun YN, et al. (2012). Miiuy croaker transferrin gene and evidence for positive selection events reveals different evolutionary patterns. PLoS One 7: e43936.

Väli Ü, Einarsson A, Waits L and Ellegren H (2008). To what extent do microsatellite markers reflect genome-wide genetic diversity in natural populations? Mol. Ecol. 17: 3808-3817.

Wang RX, Xu TJ, Sun YN and He GY (2010). Polymorphic microsatellite loci from two enriched genomic libraries for the genetic analysis of the miiuy croaker, Miichthys miiuy (Sciaenidae). Genet. Mol. Res. 9: 931-934.

Wright S (1965). The interpretation of population structure by F-statistics with special regard to systems of mating. Evolution 19: 395-420.

Xu TJ, Sun YN, Cheng YZ, Shi G, et al. (2011a). Genomic sequences comparison and differential expression of miiuy croaker MHC class I gene, after infection by Vibrio anguillarum. Dev. Comp. Immunol. 35: 483-489.

Xu TJ, Sun YN, Shi G, Cheng YZ, et al. (2011b). Characterization of the major histocompatibility complex class II genes in miiuy croaker. PLoS One 6: e23832.

Xu TJ, Sun DQ, Sun YN and Wang RX (2011c). Development of 30 novel polymorphic expressed sequence tags (EST)derived microsatellite markers for the miiuy croaker, Miichthys miiuy. Int. J. Mol. Sci. 12: 4021-4026.

Yeh F and Yang R (1997). POPGENE, the User-Friendly Shareware for Population Genetic Analysis. Molecular Biology and Biotechnology Centre, University of Alberta, Canada.

Zhang QY and Hong WS (2000). Status and prospects of artificial propagation and breeding technique of marine fish in China in the 1990s. Mod. Fish. Inf. 15: 3-6. 